Introduction to direct variational and moment methods and an application to the Child-Langmuir law

This content has been downloaded from IOPscience. Please scroll down to see the full text. 2015 Eur. J. Phys. 36065032

(http://iopscience.iop.org/0143-0807/36/6/065032)

View the table of contents for this issue, or go to the journal homepage for more

Download details:

IP Address: 129.16.69.49

This content was downloaded on 13/09/2015 at 11:59

Please note that terms and conditions apply. 


\title{
Introduction to direct variational and moment methods and an application to the Child-Langmuir law
}

\author{
D Anderson ${ }^{1}$ and M Desaix ${ }^{2}$ \\ ${ }^{1}$ Department of Space and Geophysics, Chalmers University of Technology, \\ SE-412 96 Göteborg, Sweden \\ ${ }^{2}$ School of Engineering, University College of Borås, SE-501 90 Borås, Sweden \\ E-mail: elfda@chalmers.se (D Anderson)
}

Received 2 April 2015, revised 23 July 2015

Accepted for publication 4 August 2015

Published 10 September 2015

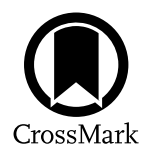

\begin{abstract}
A short introduction is given of direct variational methods and its relation to Galerkin and moment methods, all flexible and powerful approaches for finding approximate solutions of difficult physical equations. A pedagogical application of moment methods is given to the physically and technically important Child-Langmuir law in electron physics. The analysis is shown to provide simple, yet accurate, approximate solutions of the two-dimensional problem (a problem which does not allow an exact analytical solution) and illustrates the usefulness and the power of moment methods.
\end{abstract}

Keywords: direct variational methods, moment methods, Child-Langmuir law

\section{Introduction}

Variational calculus is a beautiful subject in mathematics with many applications in physics, engineering, and numerical analysis [1-6]. The essence of the variational calculus is the problem of optimizing a functional i.e. a function of a function. Variational calculus has been used during more than three hundred years since it was first formulated in order to solve the classical brachistochrone problem by Johann Bernoulli in 1696. It was then found that the optimal function must satisfy a certain differential equation, the Euler-Lagrange variational equation, directly determined by the integrand, the Lagrangian, of the functional to be optimized. However, in many cases the Euler-Lagrange equation constitutes a complicated differential equation and does not allow an explicit analytical solution. In such situations direct variational methods, e.g. the Raleigh-Ritz method based on trial functions, have been found very useful for finding approximate solutions of the problems. 
Many physically and technically important problems are formulated in terms of a differential equation. In many cases this differential equation can be reformulated as a variational problem i.e. a Lagrangian can be found such that the corresponding Euler-Lagrange variational equation is equivalent to the original equation, thus allowing the application of various variational methods, including the Raleigh-Ritz optimization method. However, it can easily be demonstrated that the characteristic equations resulting from the Raleigh-Ritz optimization procedure are in fact only special cases of the more general Galerkin and moment methods, $[6,7]$. These methods also involve the use of trial functions, but are based on the idea that certain weighted integral moments of the residue obtained by inserting the trial function into the equation should be made to vanish. An advantage of these methods as compared to the direct variational methods is that the moment methods are applicable to any differential equation.

Some applications of variational calculus, for example Hamilton's principle, are taught at the undergraduate level, but otherwise variational calculus is considered a subject more suitable for the graduate level. This is rather unfortunate since the direct variational methods developed from variational calculus, e.g. Ritz optimization, and the Galerkin and moment methods, have been widely used and provide powerful means of obtaining approximate solutions of complicated equations. It is also interesting to consider the generalization of the Raleigh-Ritz procedure to arrive at the Galerkin and moment methods. These steps, as well as the application of the different methods, can be presented in an understandable way also at the undergraduate level and can be used to encourage the curiosity and the creativity of the students. Here we have chosen to consider, as an illustrative application, the classical problem of the Child-Langmuir law in electron physics. The Child-Langmuir law determines the maximum current that can be drawn from an electron emitting surface. This problem has a simple explicit solution in the one-dimensional case. However, the two-dimensional case of a cylindrical diode has proved more difficult to solve and many ingenious procedures for finding approximate solutions have been devised during the 100 years since the law was first formulated. The purpose of the present work is to give a pedagogical demonstration of the application of the Raleigh-Ritz and Galerkin methods to a famous and well-studied physical problem and to illustrate the power and flexibility of these methods.

\section{Variational calculus, direct variational methods and the relation to Galerkin and moment methods}

Many important problems in physics and engineering can be formulated as optimization problems expressed in terms of a certain functional. Typically, this leads to a variational problem of the form

$$
\delta J[y] \equiv \delta \int_{a}^{b} L\left(x, y(x), \frac{\mathrm{d} y(x)}{\mathrm{d} x}\right) \mathrm{d} x=0,
$$

where the functional, $J[y]$, is determined by the Lagrangian $L$, for simplicity assumed to be a function of $x$, the considered function $y(x)$ and its derivative $\mathrm{d} y(x) / \mathrm{d} x$. Furthermore, assuming the function $y(x)$ to be restrained to have fixed values at the boundaries i.e. $y(a)$ and $y(b)$ are given, the variation of the functional, $\delta J[y]$, can be carried out to give

$$
\delta J[y]=\int_{a}^{b} \frac{\delta L}{\delta y} \delta y(x) \mathrm{d} x=0,
$$

where $\delta y(x)$ denotes a small deviation away from the optimal function, $y(x)$. Consequently $\delta y(a)=\delta y(b)=0$ in order to satisfy the boundary conditions on $y(x)$. The functional 
derivative of $L$, i.e. $\delta L / \delta y$, is then given by

$$
\frac{\delta L}{\delta y}=\frac{\partial L}{\partial y}-\frac{\mathrm{d}}{\mathrm{d} x} \frac{\partial L}{\partial\left(\frac{\mathrm{d} y}{\mathrm{~d} x}\right)} .
$$

Since the variation should vanish for all choices of $\delta y(x)$, this implies that the optimum function, $y(x)$, must satisfy the equation (the Euler-Lagrange variational equation)

$$
\frac{\partial L}{\partial y}-\frac{\mathrm{d}}{\mathrm{d} x} \frac{\partial L}{\partial\left(\frac{\mathrm{d} y}{\mathrm{~d} x}\right)}=0
$$

which is a second order, generally nonlinear, differential equation.

Many physically and technically important problems are formulated in terms of a differential equation $R[y]=0$. In many cases (although not always), this equation can be reformulated as a variational problem i.e. a Lagrangian, $L$, can be found such that the corresponding Euler-Lagrange variational equation is equivalent to the original equation, thus allowing the application of various variational methods. This so called inverse Lagrangian problem is discussed e.g. in [8]. However, in many cases the Euler-Lagrange equation is a complicated differential equation intractable for an explicit analytical solution. In such situations direct variational methods, e.g. the Raleigh-Ritz method, have been found very useful for finding approximate solutions of the problem. The basic idea in the Raleigh-Ritz procedure is simple, but general-instead of allowing a general variation $\delta y(x)$, the optimum solution is sought among a restricted set of trial functions of given functional form, but with flexibility incorporated by dependence on a number of parameters $\alpha_{k}$ i.e. $y(x) \rightarrow y_{T}\left(x ; \alpha_{1}, \alpha_{2}, \ldots, \alpha_{n}\right)$. This implies that the functional becomes an ordinary function of the parameters $\alpha_{k}$ and the optimization of the functional corresponds to the stationary conditions

$$
\frac{\partial J\left[y_{T}\right]}{\partial \alpha_{k}}=0, \quad k=1,2, \ldots, n,
$$

where

$$
J\left[y_{T}\right]=\int_{a}^{b} L\left(x, y_{T}(x), \frac{\mathrm{d} y_{T}(x)}{\mathrm{d} x}\right) \mathrm{d} x .
$$

Another way of writing the first variation of the functional is suggestive viz

$$
\begin{aligned}
\delta \int_{a}^{b} L\left(y_{T}\right) \mathrm{d} x & =\int_{a}^{b} \frac{\delta L\left(y_{T}\right)}{\delta y_{T}} \delta y_{T} \mathrm{~d} x \\
& =\int_{a}^{b} \frac{\delta L\left(y_{T}\right)}{\delta y_{T}}\left(\sum_{k=1}^{n} \frac{\partial y_{T}\left(x ; \alpha_{1}, \alpha_{2}, \ldots, \alpha_{n}\right)}{\partial \alpha_{k}} \delta \alpha_{k}\right) \mathrm{d} x \\
& =\sum_{k=1}^{n}\left(\int_{a}^{b} \frac{\delta L\left(y_{T}\right)}{\delta y_{T}} \frac{\partial y_{T}\left(x ; \alpha_{1}, \alpha_{2}, \ldots, \alpha_{n}\right)}{\partial \alpha_{k}} \mathrm{~d} x\right) \delta \alpha_{k}=0
\end{aligned}
$$

where we have used the fact that the variation of $y_{T}$ reduces to that of a differential with respect to the parameters $\alpha_{k}$ i.e.

$$
\delta y_{T}=\sum_{k=1}^{n} \frac{\partial y_{T}\left(x ; \alpha_{1}, \alpha_{2}, \ldots, \alpha_{n}\right)}{\partial \alpha_{k}} \delta \alpha_{k} .
$$


Since the variations of the parameters $\alpha_{k}$, i.e. $\delta \alpha_{k}$, are independent, this implies that the restricted optimization condition becomes

$$
\int_{a}^{b} \frac{\delta L\left(y_{T}\right)}{\delta y_{T}} \frac{\partial y_{T}\left(x ; \alpha_{1}, \alpha_{2}, \ldots, \alpha_{n}\right)}{\partial \alpha_{k}} \mathrm{~d} x=0, \quad k=1,2, \ldots, n
$$

which is equivalent to equation (5), but which has the advantage that it can be written as

$$
\int_{a}^{b} R\left[y_{T}\right] w_{k}(x) \mathrm{d} x=0, \quad k=1,2, \ldots, n,
$$

where $R\left[y_{T}\right]$ is the error obtained when inserting the trial function in the original equation and

$$
w_{k}(x)=\frac{\partial y_{T}\left(x ; \alpha_{1}, \alpha_{2}, \ldots, \alpha_{n}\right)}{\partial \alpha_{k}}, \quad k=1,2, \ldots, n .
$$

In this form the optimization condition implies the vanishing of certain weighted moments of the residual of the Euler-Lagrange equation when the trial function is inserted, a formulation suitable for generalization when the original equation lacks a variational reformulation. In fact, this idea is closely related to another, but even more general approximation method-the method of weighted residuals - in its simplest form called the Galerkin method [3, 4, 6]. The first part of this method is the same as in the Ritz optimization procedure: an approximate solution is sought in the form of a trial function of specified $x$-dependence but with flexibility allowed by including a number of parameters i.e. $y(x)=y_{T}\left(x ; \alpha_{1}, \alpha_{2}, \ldots, \alpha_{n}\right)$, where $y_{T}\left(x ; \alpha_{1}, \alpha_{2}, \ldots, \alpha_{n}\right)$ satisfies the boundary conditions for all parameters $\alpha_{k} ; k=1,2, \ldots, n$. Clearly this ansatz function in general does not satisfy the equation $R[y]=0$ and rather gives rise to a residual (i.e. an error) $R\left[y_{T}\left(x ; \alpha_{1}, \alpha_{2}, \ldots, \alpha_{n}\right)\right] \neq 0$. The basic idea in the method of weighted residuals is to make the absolute value of the total residual small, the total residual being defined in a weighted averaged sense by multiplying the local residual, $R\left[y_{T}\right]$, with certain weight functions $w_{k}(x)$ and integrating over the interval, i.e.

$$
\left\langle R_{k}\left[y_{T}\right]\right\rangle \equiv \int_{a}^{b} R\left[y_{T}\left(x ; \alpha_{1}, \alpha_{2}, \ldots, \alpha_{n}\right)\right] w_{k}(x) \mathrm{d} x,
$$

where $\left\langle R_{k}\left[y_{T}\right]\right\rangle$ denotes the total (integrated) residual corresponding to the weight function $w_{k}(x)$. The condition that the absolute value of $\left\langle R_{k}\left[y_{T}\right]\right\rangle$ (i.e. the total error) should be a minimum for $k=1,2, \ldots, n$ provides $n$ relations for determining the unknown parameters $\alpha_{k}$.

In fact, if the original equation allows a variational reformulation and the weight functions are taken as $w_{k}=\partial y_{T} / \partial \alpha_{k}$, the corresponding variational and Galerkin equations coincide [4, 6]. However, the Galerkin approach is more flexible than the variational approach, being applicable to a broader range of problems, e.g. problems where a variational reformulation of the original equation is not possible.

\section{The Child-Langmuir law}

A classical result in the area of electron physics is the Child-Langmuir law, which restricts the current density that can be drawn from an electron emitting surface into an evacuated diode space. The restricting force is the accumulating space charge effect, which tends to stop the electrons from leaving the surface. The original results on this problem were published already in 1911 by Child, [9], and in 1913 by Langmuir, [10] and were further improved by Langmuir and Blodgett in 1923, [11]. However, different aspects of this fundamental problem have continued to interest researchers, see e.g. [12-18]. 
The problem can be summarized as follows: consider two infinite plane parallel plates, one of which, plate A, spontaneously emits low energy electrons. Between the plates (distance $D$ ) there is a potential difference, $V_{0}$. Without loss of generality we can assume that the potential on plate $\mathrm{A}$ is zero and on the other plate, plate $\mathrm{B}$, it is $V_{0}$. If $V_{0}>0$, the electrons will be drawn towards plate $\mathrm{B}$ and there is an electron current density $i$ and a concomitant charge density $\rho$. The potential variation between the plates is given by Poisson's equation, but as long as the charge density is small, this equation can be simplified as follows

$$
\frac{\mathrm{d}^{2} V(x)}{\mathrm{d} x^{2}}=-\frac{\rho}{\epsilon_{0}} \approx 0 \rightarrow V(x)=V_{0} x / D
$$

i.e. $V(x)$ is a linear function. However, when the current (and the charge density) increases, the form of the potential changes and in particular the slope (i.e. the electric field strength) at $x=0$ decreases. Eventually the slope at $x=0$ becomes zero and larger currents cannot be drawn between the plates, since a negative potential will reflect the electrons (which are assumed to be emitted with zero energy). The magnitude of this limiting current density for given distance, $D$, and given potential jump, $V_{0}$, is determined by the Child-Langmuir law. In one dimension, this problem can easily be solved. The charge density, $\rho$, in Poisson's equation is related to the current density, $i$, and the velocity of the electrons, $v$, by the relation $i=\rho v$, where the electron velocity can be determined from the fact that the electron kinetic energy is directly related to the potential as

$$
\frac{1}{2} m v^{2}=e V
$$

Poisson's equation then becomes

$$
\begin{aligned}
& \frac{\mathrm{d}^{2} V(x)}{\mathrm{d} x^{2}}=-\frac{\rho}{\epsilon_{0}}=\frac{|i|}{\epsilon_{0}} \sqrt{\frac{m}{2 e}} \frac{1}{\sqrt{V}} \equiv \frac{K}{\sqrt{V}}, \\
& \text { where } \quad K=\frac{|i|}{\epsilon_{0}} \sqrt{\frac{m}{2 e}}
\end{aligned}
$$

Clearly the boundary conditions to go with this equation (for the limiting case) are $V(0)=0=\mathrm{d} V(0) / \mathrm{d} x$ and $V(D)=V_{0}$. The equation is consequently over-determined (three conditions on a second order differential equation) and there must be a relation between the parameters - the Child-Langmuir law.

In order to find this relation, equation (15) can be solved by first integrating once to obtain (note that $\mathrm{d} V(0) / \mathrm{d} x=0=V(0)$ )

$$
\frac{1}{2}\left(\frac{\mathrm{d} V}{\mathrm{~d} x}\right)^{2}=2 K \sqrt{V}
$$

and then once more to find the implicit solution

$$
V^{3 / 4}(x)-V_{0}^{3 / 4}=\frac{3 \sqrt{K}}{2}(x-D)
$$

which, taken at $x=0$, where $V(0)=0$, implies the Child-Langmuir law

$$
\begin{aligned}
V_{0}^{3 / 4} & =\frac{3 \sqrt{K}}{2} D \quad \rightarrow \quad|i|=\frac{4 \epsilon_{0}}{9} \sqrt{\frac{2 e}{m}} \frac{V_{0}^{3 / 2}}{D^{2}} \\
& \equiv \gamma \frac{V_{0}^{3 / 2}}{D^{2}}, \quad \text { where } \quad \gamma=\frac{4 \epsilon_{0}}{9} \sqrt{\frac{2 e}{m}} .
\end{aligned}
$$


Thus, the solution of the equation, subject to the given boundary conditions, is simply $V(x)=V_{0}(x / D)^{4 / 3}$.

The previous analysis involved in the derivation of the Child-Langmuir law was simple in the case of two infinite plane parallel plates. However, the corresponding problem in higher dimensions turns out to be analytically much more difficult and does not allow an explicit solution. Instead different analytical expansion techniques have been used to find approximate solutions for small and large distances between the surfaces and also numerical solutions have been given see e.g. [10-12]. Poisson's equation in the radially symmetric cylindrical situation reads

$$
\nabla^{2} V=\frac{1}{r} \frac{\mathrm{d}}{\mathrm{d} r}\left(r \frac{\mathrm{d} V}{\mathrm{~d} r}\right)=\frac{K}{\sqrt{V}}
$$

equation (19) is to be solved subject to the boundary conditions: $V(a)=0, V(b)=V_{0}$ and $\mathrm{d} V(a) / \mathrm{d} r=0$ where $a$ and $b$ denote the inner and outer radii respectively of the cylinder. In the low density limit $(\rho(r) \approx 0)$, the current limiting condition $(d V(a) / d r=0)$ does not apply and the corresponding solution is easily found viz. see equation (13)

$$
V(r)=V_{0} \frac{\ln (r / a)}{\ln (b / a)} .
$$

However, the full nonlinear equation describing the saturation of the electron current in the two-dimensional case has proved much more difficult and no analytical solution has been found. An ingenious transformation (see subsequent chapter) of the equation was introduced by Langmuir already in 1913 and this has served as a starting point for many subsequent investigations.

\section{The Langmuir beta equation}

Poisson's equation in the radially symmetric cylindrical case, equation (19), can be reformulated as the variational problem corresponding to the Lagrangian

$$
L=\frac{1}{2} r\left(\frac{\mathrm{d} V}{\mathrm{~d} r}\right)^{2}+2 r K \sqrt{V}
$$

This could serve as a starting point for an investigation using direct variational methods. However, Langmuir, in his first paper on the subject, [10], aimed directly at a 2D formulation of the Child-Langmuir law. To this end he introduces a new function, Langmuir's betafunction, $\beta(x)$ (here denoted $y(x))$ and a new more convenient variable $x=\ln (r / a)$, see equation (20), and writes the Child-Langmuir law as

$$
|i(r)|=\frac{4 \epsilon_{0}}{9} \sqrt{\frac{2 e}{m}} \frac{V^{3 / 2}}{r^{2} y^{2}},
$$

where the (non-dimensional) function $y=y(x)$ accounts for the 2D modifications of the 1D Child-Langmuir law. Introducing this ansatz into Poisson's equation and using the fact that $j=2 \pi r i$ (the current density per unit length in the axial direction) is independent of $r$, it is possible to transform the equation for $V(r)$ into the following nonlinear differential equation for $y(x)$ : 


$$
R[y] \equiv 3 y \frac{\mathrm{d}^{2} y}{\mathrm{~d} x^{2}}+\left(\frac{\mathrm{d} y}{\mathrm{~d} x}\right)^{2}+4 y \frac{\mathrm{d} y}{\mathrm{~d} x}+y^{2}-1=0 .
$$

The boundary conditions on $y(x)$ are: $y(0)=0$ and $y(x) \rightarrow 1$ as $x \rightarrow \infty$. We also note that $\mathrm{d} y(0) / \mathrm{d} x=1$ (actually as a direct consequence of the equation). In fact, for small values of $x$, the beta function varies as $y(x) \approx x$, which implies that equation (22) reduces to the $1 \mathrm{D}$ result where $r-a<b-a=D \ll a$ and radial effects are negligible.

Langmuir's beta equation is a very complicated equation and no analytical solution has been found. A large number of investigations based on equation (23) see [10-12] has been done over the years. Analytical solutions have been found in terms of expansions both for small and large values of the parameter $x$ and numerical solutions have also been given. In fact only as recently as in 2004 was a closed form approximate analytical solution found for the potential variation in the $2 \mathrm{D}$ case [17] and shown to give good agreement with previous approximate and numerical results. In the present work we will, using moment methods, present simple explicit approximations for $y(x)$ instead of the complicated expansions suggested by Langmuir (for small values of $x$ ) and later by Page and Adams, [12] (for large values of $x$ ).

A reformulation of equation (23) in terms of a variational problem does not seem possible. However, the various moment methods discussed in a previous section are easily applicable. The first problem is to choose a proper trial function. The choice is made based on different information available for the problem e.g. asymptotic analysis in complementary limits. In the present problem the requirement is that it should go smoothly from zero to unity as the independent variable goes from zero to infinity. Clearly this leaves a wide range of acceptable trial functions. A further restraint is that the concomitant evaluation of the moments should be possible to perform analytically. A possible choice is the smooth function $y_{T}(x ; \alpha)=\tanh (\alpha x)$. Strictly speaking, this equation does not satisfy the initial condition on the derivative i.e. $\mathrm{d} y(0) / \mathrm{d} x=1$ (unless $\alpha=1$ ). However, this feature (the correct 1D limit) is here sacrificed for the benefit of flexibility and good overall agreement in the 2D domain. The next step is to choose the weight function. If the beta equation had been derived from a variational problem, the Raleigh-Ritz procedure would have prescribed the weight function $w_{T}(x)=\partial y_{T}(x ; \alpha) / \partial \alpha=x \operatorname{sech}^{2}(\alpha x)$. When this trial function and the correspondingly chosen weight function are used to evaluate the moment, one obtains the total residual

$$
\begin{aligned}
\left\langle R\left[y_{T}\right]\right\rangle & =\int_{0}^{\infty} R\left[y_{T}(x ; \alpha)\right] w_{T}(x) \mathrm{d} x \\
& =7 J_{6}-6 J_{4}+\frac{2}{3 \alpha}-\frac{J_{4}}{\alpha^{2}}
\end{aligned}
$$

where $J_{n}=\int_{0}^{\infty} x \operatorname{sech}^{n} x \mathrm{~d} x$ and in particular

$$
J_{4}=\frac{2}{3} \ln 2-\frac{1}{6}, \quad J_{6}=\frac{8}{15} \ln 2-\frac{11}{60} .
$$

Ideally, the value of $\alpha$ should be determined by the condition $\left\langle R\left[y_{T}\right]\right\rangle=0$. However, the corresponding equation does not have a real solution for $\alpha$ since $\left\langle R\left[y_{T}\right]\right\rangle<0$ for all $\alpha$. The best one can do then is to minimize the absolute error $\left|\left\langle R\left[y_{T}\right]\right\rangle\right|$ (note that $\left\langle R\left[y_{T}\right]\right\rangle<0$ ). The concomitant condition $\left(\mathrm{d}\left\langle R_{T}(y)\right\rangle / \mathrm{d} \alpha=0\right)$ yields $\alpha=3 J_{4} \approx 0.886$. It could be noted that this value implies an initial slope close to the value, $\alpha=1$, that gives exact agreement with the $1 \mathrm{D}$ result. The overall agreement with the numerically obtained $2 \mathrm{D}$ solution is found to be good, see figure 1, in spite of the simplicity of the solution. 


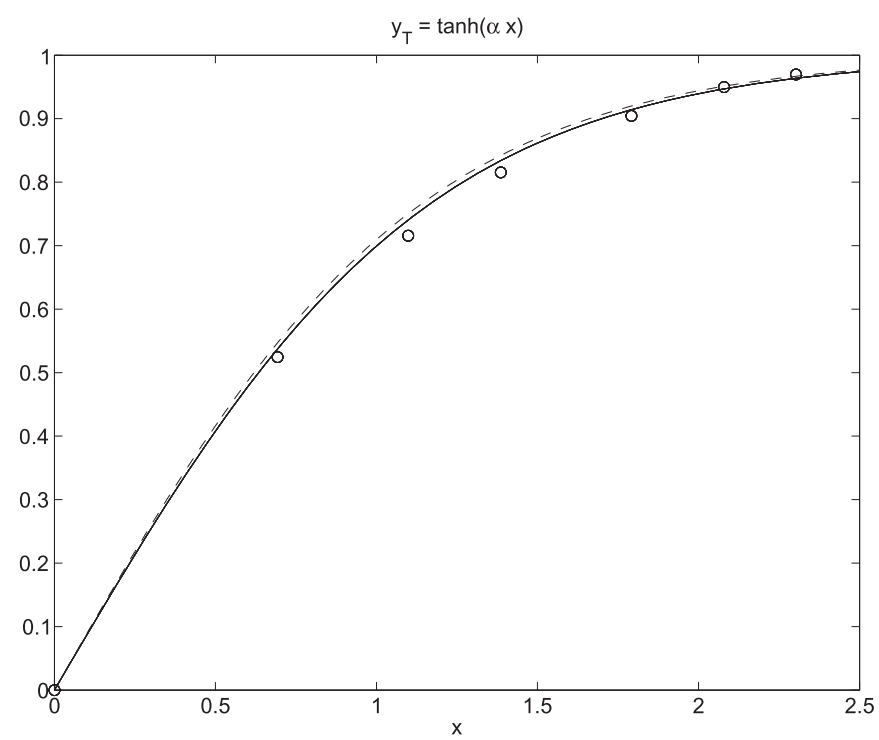

Figure 1. Comparison between the second approximation $y_{T}=\tanh (x \sqrt{3} / 2)$ (solid) and $y_{T}=\tanh (0.886294 x)$ (dashed line) and tabulated values for $y$ (circles).

A simple modification of the solution is worth mentioning: the chosen weight function $\left(w_{T}(x)=x \operatorname{sech}^{2}(\alpha \mathrm{x})\right)$ tends to suppress residual errors in the equation for small and large values of $x$. Using instead the 'neutral' weight function $w_{T}(x)=1$, the corresponding total residual becomes $\left\langle R\left[y_{T}\right]\right\rangle=2-4 \alpha / 3-1 / \alpha<0$, which upon maximization, i.e. minimization of the absolute error, gives $\alpha=\sqrt{3} / 2 \approx 0.866$ i.e. close to the previously found value. The agreement with the numerical solution is again good, in fact even better than the previous approximation, see figure 1 . This illustrates the fact that the quality of a certain trial function and a concomitant weight function is very difficult to assess a priori.

\section{Conclusion}

The present work provides a short pedagogical introduction to direct variational methods and the further development to general moment methods. The moment approach is illustrated by an application to the Child-Langmuir law in two dimensions, a problem which has eluded analytical solutions for a hundred years and which has attracted much interest and effort over these years. The approximate solutions found here for this problem illustrate the usefulness of the moment methods and provide simple, yet accurate, approximations.

\section{References}

[1] Fox C 1954 An Introduction to the Calculus of Variations (Oxford: Oxford University Press)

[2] Gelfand I M and Fomin S V 1963 Calculus of Variations (Englewood Cliffs, NJ: Prentice-Hall)

[3] Wan F Y M 1995 Introduction to the Calculus of Variations and Its Applications (New York: Chapman \& Hall)

[4] Komzsik L 2009 Applied Calculus of Variations for Engineers (Boca Raton, FL: CRC Press)

[5] Vujanovic B D and Jones S E 1989 Variational Methods in Nonconservative Phenomena (Boston, MA: Academic) 
[6] Finlayson B A 1972 The Method of Weighted Residuals and Variational Principles (New York: Academic)

[7] Reddy J N 1986 Applied Functional Analysis and Variational Methods in Engineering (New York: McGraw-Hill)

[8] Santilli R M 1978 Foundations of Theoretical Mechanics: 1. The Inverse Problem in Newtonian Mechanics (Berlin: Springer)

[9] Child C D 1911 Discharge from hot CAO Phys. Rev. 32498

[10] Langmuir I 1913 The effect of space charge and residual gases on thermionic currents in high vacuum Phys. Rev. Second Ser. 2 450-86

[11] Langmuir I and Blodgett K 1923 Currents limited by space charge between coaxial cylinders Phys. Rev. 22347

[12] Page L and Adams N I Jr 1943 Space charge between coaxial cylinders Phys. Rev. 68126

[13] Luginsland J W, Lau Y Y and Gilgenbach R M 1996 Two-dimensional Child-Langmuir law Phys. Rev. Lett. 774668

[14] Umstadt R J and Luginsland J W 2001 Two-dimensional space-charge-limited emission: beam edge characteristics and applications Phys. Rev. Lett. 87145002

[15] Lau Y Y 2001 Simple theory for the two-dimensional Child-Langmuir law Phys. Rev. Lett. 87 278301

[16] Watrous J J, Luginsland J W and Frese M H 2001 Current and current density of a finite-width, space-charge-limited electron beam in two-dimensional, parallel-plate geometry Phys. Plasmas 84202

[17] Chen X, Dickens J, Hatfield L L, Choi E-H and Kristiansen M 2004 Approximate analytical solutions for the space-charge-limited current in one-dimensional and two-dimensional cylindrical diodes Phys. Plasmas 113278

[18] Mahalingam S, Nieter C, Loverich J, Smithe D and Stoltz P 2009 Space charge limited currents calculations in coaxial cylindrical diodes using particle-in-cell simulations Open Plasma Phys. J. 263 